\title{
Characteristics of Head Center of Mass Sway and Neck Flexor Activity During Compensatory Backward Stepping in the Elderly
}

\author{
Yahiko Takeuchi ${ }^{1 *}$, Kimiya Fujio ${ }^{2}$ and Ryo Fukata ${ }^{3}$ \\ ${ }^{1}$ Department of Physical Therapy, Josai International University, 1 Gumyo, Togane, Chiba, 283-8555, Japan \\ ${ }^{2}$ Department of Rehabilitation for the Movement Functions, Research Institute of the National Rehabilitation, Center for Persons \\ with Disabilities, 1-4 Namiki, Tokorozawa, Saitama, 359-8555, Japan
}

${ }^{3}$ Department of Rehabilitation, Chiba University Hospital, 1-8-1 Inohana, Chuoku, Chiba, 260-8677, Japan

*Corresponding author: Yahiko Takeuchi, Department of Physical Therapy, Josai International University, 1 Gumyo, Togane, Chiba, 283-8555, Japan

\section{ARTICLE INFO}

Received: 慧 August 23, 2021

Published: 幽 August 31, 2021

Citation: Yahiko Takeuchi, Kimiya Fujio, Ryo Fukata. Characteristics of Head Center of Mass Sway and Neck Flexor Activity During Compensatory Backward Stepping in the Elderly. Biomed J Sci \& Tech Res 38(3)-2021. BJSTR. MS.ID.006157.

Keywords: Compensatory Stepping; Elderly; Head Center of Mass; Sternocleidomastoid Activity

Abbreviations: COM: Center of Mass; EMG: Electromyography; SCM: Sternocleidomastoid Muscle; QS: Quiet Standing; PS: Perturbation Stepping; SS: Single Stance; MVC: Maximum Voluntary Contraction; ANOVA: Analysis of Variance

\section{Abstract}

Research Question: This study aimed to determine age-related changes in head control during compensatory stepping against postural perturbation.

Methods: Fifteen community-dwelling elderly individuals and 11 young adults participated in this study. We carried out the Push and Release Test, wherein a participant leaned on to the examiner's hands. A compensatory backward stepping was triggered by suddenly releasing the supporting hands. Based on both ground reaction force and acceleration of the examiners' hands, backward stepping was divided into four phases. Head sway and activity of sternocleidomastoid muscle were measured using a three-dimensional motion analysis system and an electromyograph. We determined the relationship between age and stepping phase in terms of head sway and sternocleidomastoid activity.

Results: The results of a two-way analysis of variance confirmed the relationship between age and stepping phase in the amount of anteroposterior sway of head center of mass. T-test results revealed that, due to the sternocleidomastoid activity, the elderly group had significantly higher values in the single-stance phase than the young-adult group.

Significance: Our data indicate a change with age in the compensatory stepping in response to load disturbances due to a decline of the ability of an individual to orient the center of mass of the head to an optimal location during compensatory stepping. In addition to insufficiencies of sensory inputs, the overactivity of the sternocleidomastoid is considered as one of the mechanisms for head control deficits.

\section{Introduction}

Falls in elderly people are among the major problems in the aging society. It causes serious injuries, such as a vertebral fracture and head trauma, inducing health-related adverse consequences. Previous studies have reported on the risk factors of falls and preventive strategies for elderly people $[1,2]$. It is proposed that a primary factor of falls was the decline in standing balance, both static and dynamic postural control [3]. In general, there are three different strategies to compensate for imbalance, which are as follows: ankle, hip, and stepping strategies [4]. Among these postural strategies, the stepping strategy, the compensation for lost 
of balance with a single step, is the last bastion to prevent falls [5] In addition, elderly individuals tend to frequently lose their balance in the backward direction due to the kyphotic deformity in the thoracic spine [6-8]. Therefore, it is crucial for elderly people to be capable of backward stepping against balance loss.

The human body has a complex segmented structure, which is composed of the head, trunk, and four limbs. The center of mass (CoM) in the frontal/sagittal plane of each segment is not uniform [9]. Correspondingly, this location must be perceived, even during postural control strategies such as the stepping response, and analyzing the characteristics of positional changes (i.e., perturbations) is thought to be important. Particularly, with the weight of a head being approximately $7 \%$ of the total body weight [10] and its range of motion positioned superior to the cervical vertebrae, the head CoM is believed to have a significant influence on the COM of a whole body. Head stabilization is necessary to optimize conditions for the visual organs and maintaining head stability may be one of the most fundamental tasks of the postural control system $[11,12]$. On the other hand, there are no reports regarding the characteristics of the sway of head CoM in compensatory backward stepping. We assumed that head control in distinctive phases of stepping is one of the important factors for fall prevention. Therefore, this study aimed to determine the agerelated changes in head control, based on the characteristics of the sway of the head CoM during compensatory stepping due to a load disturbance.

\section{Methods}

\section{Participants}

The study participants were 15 healthy elderly people living in the community ( 6 women, 9 men; average age, $66.7 \pm 3.0$ years) and 11 young adults ( 4 women, 7 men; average age, $20.5 \pm 1.4$ years). All participants received explanations about the purpose of the study and experimental procedure, and provided written informed consent. This study was approved by the ethics committee of the Chiba Prefectural University of Health Sciences, which is the former institution of the first author.

\section{Measurements and Experimental Procedure}

Ground reaction forces were recorded from 4 force plates with a sampling frequency at $1,000 \mathrm{~Hz}$ (AMTI, BP400600). One pair of force plates was used for the starting position and the other pair was used for the reaction force by the landing limb. The present study focused on age-related changes of compensatory backward stepping to recovery from balance loss. To evoke backward stepping, the Push and Release Test was adopted [13,14]. In this test, the participant was instructed to stand on the pair of force plates and to lean on to the examiner's hands, which supported the participant's upper back. Then, the examiner suddenly released his hands and so, the participant stepped backward to maintain standing balance. The release timing of the examiners' hands was defined as the moment when the midpoint of the putative line between the acromion and greater trochanter of the participant was over the posterior edge of heels. It was determined by a visual inspection through a large mirror, and identified with the use of an accelerometer attached on the back of the examiner's hands [15]. The accelerometer was digitized at $1,000 \mathrm{~Hz}$.

To measure the head movement during backward stepping, kinematics data were recorded using a 3D motion capture system (Motion Analysis Co., Mac3D, California, USA). Twenty-nine infrared reflective markers were placed based on the Helen Hayes marker set [16]. Kinematic signals from the motion capture system camera were sampled at $200 \mathrm{~Hz}$. We further performed Electromyography (EMG) of the Sternocleidomastoid Muscle (SCM), which is involved in head control during backward stepping. The EMG electrodes (S\&ME Co., DL-141, Tokyo, Japan) were attached on the sternal head of the SCM at the level of the thyroid cartilage. We measured the unilateral musculus SCM to confirm that the rotation movement of the cervical vertebrae did not occur before the task movement. The EMG signal was recorded at $1,000 \mathrm{~Hz}$ and through a band-pass filter (55-500 Hz) to decrease movement artifact and extraneous noise.

\section{Data Analysis}

Based on a vertical component of ground reaction forces, compensatory backward stepping was divided into the following four phases (Figure 1): Quiet Standing (QS), Perturbation Stepping (PS) Single Stance (SS), and landing. PS was defined as the moment from the onset of hands release to the time when the stepping limb was removed from the force plate. Time window for analysis was $1.0 \mathrm{~s}$ for QS and $0.1 \mathrm{~s}$ for landing. For the data analysis, the displacement of head CoM was defined by the standard deviation of locational data at each camera angle for each phase of backward compensatory stepping. The EMG activity in the SCM was normalized using \% Maximum Voluntary Contraction (MVC) at $100 \%$. A two-way analysis of variance (ANOVA) was performed on head CoM and SCM activity, with age group and stepping phase used as factors. Post hoc analysis was Comparisons between age groups and stepping phases were made using an unpaired t-test and a multiple comparison test, respectively. The significance level was set at $5 \%$. 


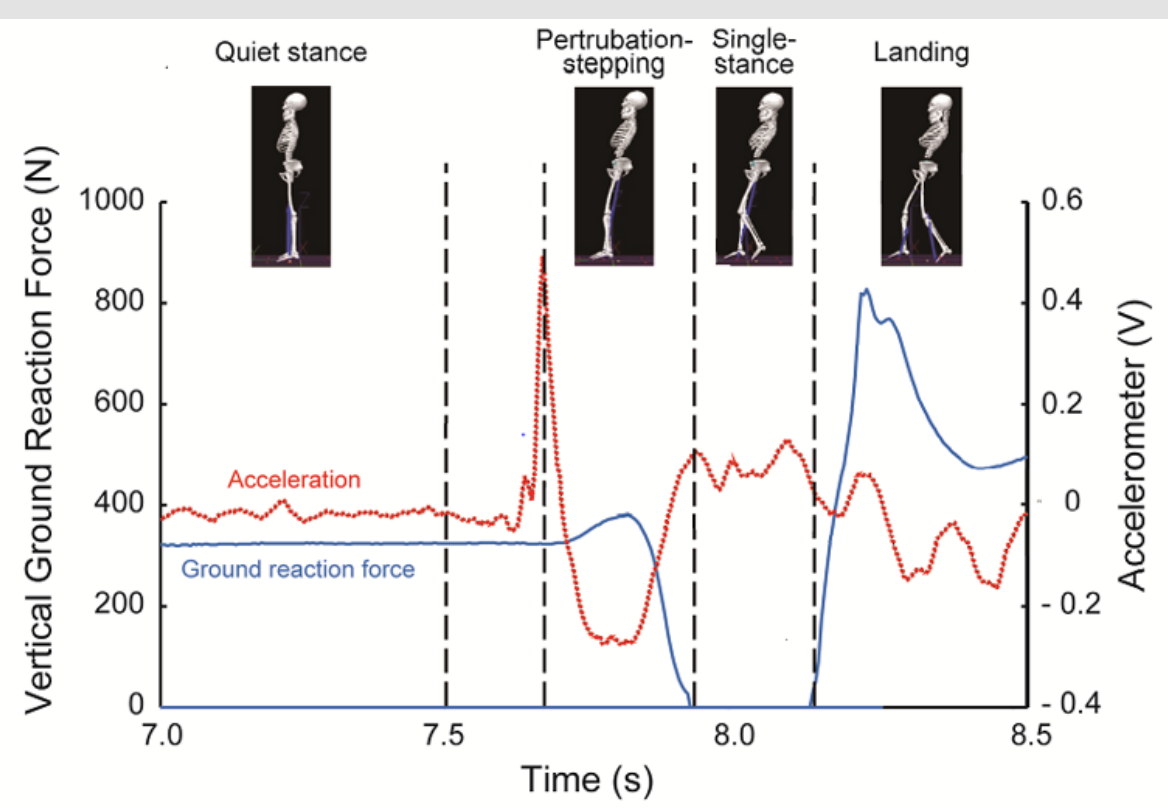

Figure 1: Phase separation of compensatory backward stepping based on disturbance acceleration and ground reaction force.

\section{Results}

Table 1 shows the amount of head COM sway in each phase of compensatory backward stepping. The two-way ANOVA showed a significant main effect on phase $(\mathrm{F}=49.7, \mathrm{p}<0.01)$, and the interaction between age and phase in the amount of anteroposterior sway of the head COM (F=4.41, p<0.01). Figure 2 shows the correlation between age group and stepping phase of compensatory backward stepping. Regarding SCM activity, no interaction was observed between the two factors, but a main effect was observed for both age group and stepping phase factors. The results of the t-test for the age group factor revealed that the elderly group had significantly higher values in the SS phase than the young-adult group (Figure 3). Moreover, the results of the multiple comparison test for the stepping phase factor indicated that the elderly group had significantly higher values during the PS, SS, and landing phases than during the QS phase (Figure 3). However, the young-adult group showed no significant differences in any phase.

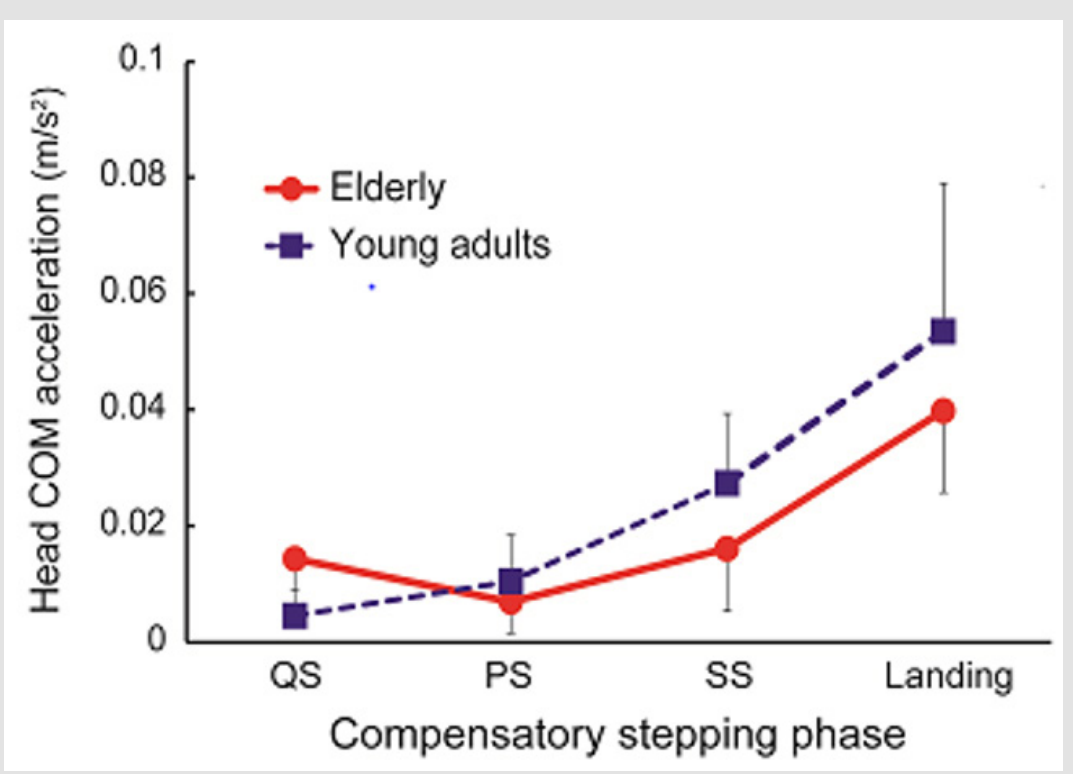

Figure 2: The interaction between age group and stepping phase in compensatory backward stepping. Note: COM, Center of Mass. 


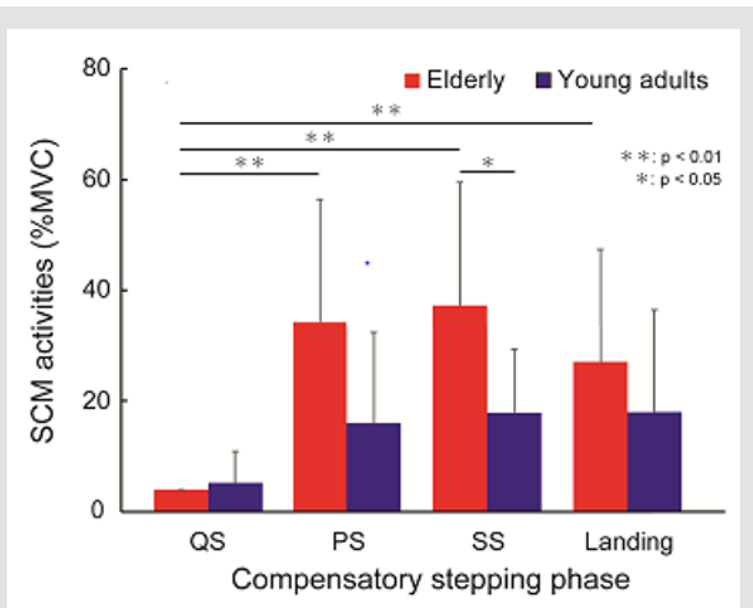

Figure 3: The results of the t-test for the age group factor and the multiple comparison test for the stepping phase factor in the amount of sternocleidomastoid muscle activity during compensatory backward stepping.

Note: SCM, sternocleidomastoid muscle.

Table 1: The amount of head center of mass sway in each phase of compensatory backward stepping.

\begin{tabular}{|c|c|c|c|c|c|}
\hline & & QS & PS & SS & Landing \\
\hline A-P & Elderly & $0.014 \pm 0.011$ & $0.007 \pm 0.005$ & $0.016 \pm 0.011$ & $0.040 \pm 0.014$ \\
\hline & Young & $0.005 \pm 0.004$ & $0.010 \pm 0.008$ & $0.027 \pm 0.012$ & $0.054 \pm 0.025$ \\
\hline M-L & Elderly & $0.002 \pm 0.001$ & $0.003 \pm 0.002$ & $0.013 \pm 0.007$ & $0.013 \pm 0.004$ \\
\hline & Young & $0.001 \pm 0.001$ & $0.002 \pm 0.001$ & $0.01 \pm 0.08$ & $0.017 \pm 0.008$ \\
\hline
\end{tabular}

Note: $\left(\right.$ mean $\left.\pm \mathrm{SD}, \mathrm{m} / \mathrm{s}^{2}\right)$

A-P, anterior-posterior; M-L, medial-lateral; QS, quiet standing; PS, perturbation-stepping; SS, single stance; SD, standard deviation.

\section{Discussion}

The results of the present study showed a smaller posterior displacement of head COM, with a larger sway in the standing phase, in the elderly group than in the young-adult group. On the other hand, the SCM activity that contributes to head movements was larger in the elderly group than in the young-adult group, although the difference was not significantly different in the standing phase. These results imply that the head control strategy in the anteroposterior direction varies depending on age; elderly people have increased stiffness due to the hyperactivation of the neck muscles, rather than flexible coordination. One of the possible mechanisms is the restriction of neck joint movement due to higher muscle tonus and structural changes of the neck joint. The cervical spine has the greatest mobility in the human vertebrae, which stabilizes the visual and vestibular organs in space during spatial localization [17]. In general, aging increases the muscle tone of the shoulder girdle and neck joints [18], causing restriction in the range of motion in the cervical and thoracic spine. Reduced joint movement would affect the consolidation of information from the sensory organs and deteriorate both static and dynamic head control [19].
This explanation is supported by the SCM activity, wherein the agonist muscle of neck flexion compensates for the backward postural disturbance. The elderly stabilize their head position with pronounced SCM activity throughout each successive phase of backward compensatory stepping (i.e., from QS to landing). In particular, the SCM activity in the SS phase was significantly higher in the elderly group than in the young-adult group, which drastically reduces the base of support. Apart from their role in flexible joint coordination, the activities of larger neck muscles compensate to stabilize the head position on the same location. The activity of the SCM is known to be evoked by the startle reflex [20]. The present perturbation paradigm, which states that a rapid backward perturbation can evoke the startle reflex, may be pronounced in elderly people. A limitation of our study is its small sample size, as only a few participants were recruited due to the risk of disturbance loading. In addition, this study was conducted to investigate the effects of aging by comparing the data between the elderly and young-adult participants. It is necessary to investigate in the future the differences between men and women, because sex differences in postural regulatory mechanisms have been reported in the aging process [21] 


\section{Conclusion}

The results of the present study revealed that the compensatory stepping of elderly people against a load disturbance involves smaller anteroposterior sway of the head CoM compared to that of young adults. This indicated age-related changes in the compensatory stepping against load disturbances, as the elderly population has a reduced ability to optimize the spatial localization of the head through the consolidation of sensory organ information. The results also indicated the activation of the SCM, the principal agonist of neck flexion, as one of the mechanisms controlling the head CoM during compensatory stepping.

\section{Conflict of Interest Statement}

None declared.

\section{Author Contributions}

- Yahiko Takeuchi: Conceptualization, Methodology, Software, Formal analysis, Writing-Original draft preparation, Project administration, and Funding acquisition.

- Kimiya Fujio: Methodology, Software, Visualization, and Writing-Review and Editing.

- $\quad$ Ryo Fukata: Writing-Review and Editing.

\section{Data Statement}

All data are fully available without restriction.

\section{Acknowledgment}

This work was supported by JSPS KAKENHI Grant Number 17 K07594.

\section{References}

1. Currie L (2008) Fall and injury prevention. In: RG Hughes (Edt.)., Patient Safety and Quality: An Evidence-Based Handbook for Nurses, Agency for Healthcare Research and Quality (US), Rockville.

2. Lukaszyk C, Harvey L, Sherrington C, Keay L, Tiedemann A, et al. (2016) Risk factors, incidence, consequences and prevention strategies for falls and fall-injury within older indigenous populations: a systematic review. Aust N Z J Public Health 40(6): 564-568.

3. Berg K, Wood-Dauphine S, Williams JI, Gayton D (1989) Measuring balance in the elderly: preliminary development of an instrument. Physiother Can 41(6): 304-311.

4. Horak FB, Shupert CL, Mirka A (1989) Components of postural dyscontrol in elderly: a review. Neurobiol Aging 10(1989): 727-738.

5. Maki BE, McIlroy WE (2006) Control of rapid limb movements for balance recovery: age-related changes and implications for fall prevention. Age Aging 35(2): ii12-ii18.

6. Elble RJ (1997) Change in gait with normal aging. In: JC Masdeu, Sudarsky, Wolfson (Eds.)., Gait Disorders of Aging ( $1^{\text {st }}$ Edn.)., LippincottRaven, Philadelphia, pp. 93-105.

7. Kasukawa Y, Miyakoshi N, Hongo M, Ishikawa Y, Noguchi H, et al. (2010) Relationships between falls, spinal curvature, spinal mobility and back extensor strength in elderly people. J Bone Miner Metab 28(1): 82-87.

8. Sran MM, Robinovitch SN (2008) Preventing fall-related vertebral fractures: effect of floor stiffness on peak impact forces during backward falls. Spine (Phila Pa 1976) 33(17): 1856-1862.

9. Winter DA (2009) Biomechanics and Motor Control of Human Movement ( $4^{\text {th }}$ Edn.)., John Wiley \& Sons, New Jersey.

10. Judovich BD (1952) Herniated cervical disc; a new form traction therapy. Am J Surg 84(1952): 646-656.

11. Nicholas SC, Doxey-Gasway DD, Paloski WH (1998) A link-segment model of upright human posture for analysis of head-trunk coordination. J Vestib Res 8(3): 187-200.

12. Horak FB (2006) Postural orientation and equilibrium: what do we need to know about neural control of balance to prevent falls? Age Ageing 35(2): ii7-ii11.

13. Jacobs JV, Horak FB, Van Tran K, Nutt JG (2006) An alternative clinical postural stability test for patients with Parkinson's disease. J Neurol 253(11): 1404-1413.

14. Horak FB, Wrisley DM, Frank J (2009) The Balance Evaluation Systems Test (BESTest) to differentiate balance deficits. Phys Ther 89(5): 484498.

15. Takeuchi Y (2018) A successful backward step correlates with hip flexion moment of supporting limb in elderly people. PLoS One 13(1): e0190797.

16. Kadaba MP, Ramakrishnan HK, Wootten ME (1990) Measurement of lower extremity kinematics during level walking. J Orthop Res 8(3): 383-392.

17. Neumann DA (2010) Kinesiology of the Musculoskeletal System. $\left(2^{\text {nd }}\right.$ Edn.)., Mosby, St. Louis.

18. Kocur P, Grzeskowiak M, Wiernicka M, Goliwas M, Lewandowski J, et al. (2017) Effects of aging on mechanical properties of sternocleidomastoid and trapezius muscle during transition from lying to sitting position -a cross-sectional study, Arch Gerontol Geriatr 70: 14-18.

19. Toledo DR, Barela JA (2010) Sensory and motor differences between young and older adults: somatosensory contribution to postural control. Rev Bras Fisioter 14(3): 267-275.

20. Sanders O, Hsiao HY, Savin DN, Creath RA, Rogers MW (2019) Aging changes in protective balance and startle responses to sudden drop perturbations. J Neurophysiol 122(1): 39-50.

21. Sullivan EV, Rose J, Torsten R, Pfefferbaum A (2009) Postural sway 
ISSN: 2574-1241

DOI: 10.26717/BJSTR.2021.38.006157

Yahiko Takeuchi. Biomed J Sci \& Tech Res

(C) (P) This work is licensed under Creative

Submission Link: https://biomedres.us/submit-manuscript.php

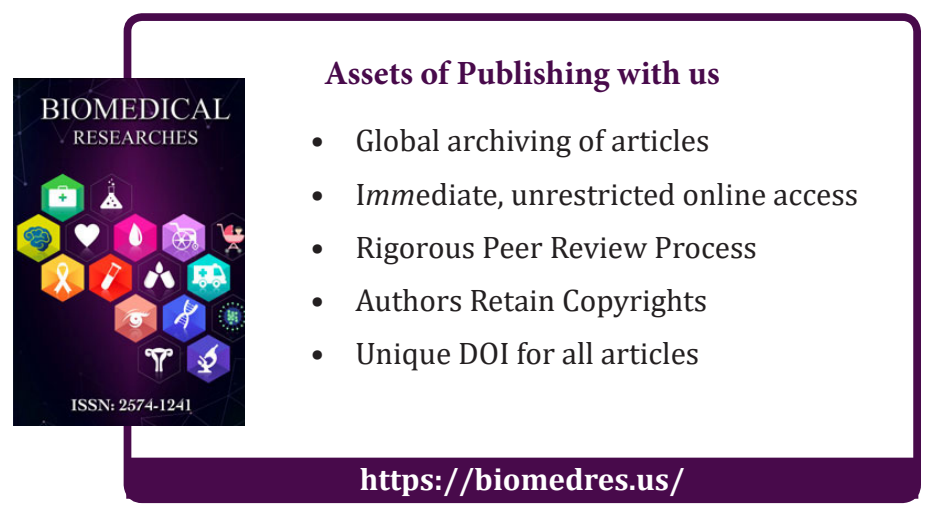

